Electronic Supplementary Information

\title{
Semicoordination Bond Breaking and Halogen Bond Making Changes the Supramolecular Architecture of Metal-containing Aggregates
}

Lev E. Zelenkov, ${ }^{1,2}$ Daniil M. Ivanov, ${ }^{1}$ Evgeniy K. Sadykov, ${ }^{3}$ Nadezhda A. Bokach,,${ }^{1 *}$ Bartomeu Galmés, ${ }^{4}$ Antonio Frontera ${ }^{4}$ and Vadim Yu. Kukushkin ${ }^{1,5}$

${ }^{1}$ Institute of Chemistry, Saint Petersburg State University, Universitetskaya Nab. 7/9, Saint Petersburg, 199034 Russian Federation

${ }^{2}$ Department of Physics and Engineering, ITMO University, St. Petersburg, 197101, Russia ${ }^{3}$ A.E. Favorsky Irkutsk Institute of Chemistry SB RAS, Favorsky St. 1, Irkutsk, 664033 Russian Federation

${ }^{4}$ Department of Chemistry, Universitat de les Illes Balears, Crta de Valldemossa km 7.5, 07122 Palma de Mallorca (Baleares), Spain

${ }^{5}$ Laboratory of Crystal Engineering of Functional Materials, South Ural State University, 76, Lenin Av., Chelyabinsk, 454080 Russian Federation 
Table S1. Crystallographic data and structure refinement for $\mathbf{1} \cdot 2(1,4-\mathrm{FIB})$ and $\mathbf{1} \cdot 2(1,3,5-\mathrm{FIB})$.

\begin{tabular}{|c|c|c|}
\hline Identification code & 1.2(1,4-FIB) & $\mathbf{1} \cdot 2(1,3,5-\mathrm{FIB})$ \\
\hline CCDC number & 2016545 & 2016547 \\
\hline Empirical formula & $\mathrm{C}_{18} \mathrm{H}_{10} \mathrm{~F}_{8} \mathrm{I}_{4} \mathrm{NiO}_{2} \mathrm{~S}_{4}$ & $\mathrm{C}_{18} \mathrm{H}_{10} \mathrm{~F}_{6} \mathrm{I}_{6} \mathrm{NiO}_{2} \mathrm{~S}_{4}$ \\
\hline Formula weight & 1104.81 & 1320.61 \\
\hline Temperature/K & $100(2)$ & $100(2)$ \\
\hline Crystal system & monoclinic & monoclinic \\
\hline Space group & $\mathrm{P} 2_{1} / \mathrm{c}$ & $\mathrm{P} 2_{1} / \mathrm{n}$ \\
\hline $\mathrm{a} / \AA$ & $4.44780(10)$ & $4.3932(6)$ \\
\hline $\mathrm{b} / \AA$ & $11.1329(3)$ & $13.0695(19)$ \\
\hline $\mathrm{c} / \AA$ & $28.6259(9)$ & $27.006(4)$ \\
\hline$\alpha / /^{\circ}$ & 90 & 90 \\
\hline$\beta /{ }^{\circ}$ & $90.373(3)$ & $91.815(14)$ \\
\hline$\gamma /{ }^{\circ}$ & 90 & 90 \\
\hline Volume $/ \AA^{3}$ & $1417.44(7)$ & $1549.8(4)$ \\
\hline$Z$ & 2 & 2 \\
\hline$\rho_{\text {calc }} \mathrm{g} / \mathrm{cm}^{3}$ & 2.589 & 2.830 \\
\hline$\mu / \mathrm{mm}^{-1}$ & 38.613 & 6.925 \\
\hline $\mathrm{F}(000)$ & 1020.0 & 1196.0 \\
\hline Crystal size $/ \mathrm{mm}^{3}$ & $0.21 \times 0.18 \times 0.15$ & $0.21 \times 0.19 \times 0.17$ \\
\hline Radiation & $\operatorname{CuK} \alpha(\lambda=1.54184)$ & $\operatorname{MoK} \alpha(\lambda=0.71073)$ \\
\hline $2 \Theta$ range for data collection ${ }^{\circ}$ & 6.176 to 141.17 & 6.234 to 63.166 \\
\hline Index ranges & $\begin{aligned}-5 & \leq \mathrm{h} \leq 4, \\
-13 & \leq \mathrm{k} \leq 13, \\
-34 & \leq 1 \leq 34\end{aligned}$ & $\begin{aligned}-6 & \leq \mathrm{h} \leq 5 \\
-19 & \leq \mathrm{k} \leq 18 \\
-38 & \leq 1 \leq 39\end{aligned}$ \\
\hline Reflections collected & 7082 & 18144 \\
\hline Independent reflections & $\begin{array}{l}2694 \\
{\left[R_{\text {int }}=0.0493\right.} \\
\left.R_{\text {sigma }}=0.0517\right]\end{array}$ & $\begin{array}{l}4672 \\
{\left[R_{\text {int }}=0.0729,\right.} \\
\left.R_{\text {sigma }}=0.0686\right]\end{array}$ \\
\hline Data/restraints/parameters & $2694 / 0 / 170$ & $4672 / 0 / 170$ \\
\hline Goodness-of-fit on $\mathrm{F}^{2}$ & 1.008 & 1.043 \\
\hline Final R indexes $[\mathrm{I} \geq 2 \sigma(\mathrm{I})]$ & $\begin{array}{l}\mathrm{R}_{1}=0.0469 \\
\mathrm{wR}_{2}=0.1222\end{array}$ & $\begin{array}{l}\mathrm{R}_{1}=0.0428 \\
\mathrm{wR}_{2}=0.0720\end{array}$ \\
\hline Final R indexes [all data] & $\begin{array}{l}\mathrm{R}_{1}=0.0530 \\
\mathrm{wR}_{2}=0.1279\end{array}$ & $\begin{array}{l}\mathrm{R}_{1}=0.0634 \\
\mathrm{wR}_{2}=0.0790\end{array}$ \\
\hline Largest diff. peak/hole / e $\cdot \AA^{-3}$ & $1.82 /-1.61$ & $1.32 /-1.84$ \\
\hline
\end{tabular}


Table S2. Selected bond lengths $(\AA)$ and angles $\left(^{\circ}\right)$ in the cocrystals of $\mathbf{1} \cdot 2(1,4-\mathrm{FIB})$ and 1 $2(1,3,5$-FIB $)$.

\begin{tabular}{lcl}
\hline Bond/angle & $\mathbf{1 \cdot 2}(1,4-\mathrm{FIB})$ & $\mathbf{1 \cdot 2}(1,3,5-\mathrm{FIB})$ \\
\hline Ni1-S1 & $2.2221(16)$ & $2.2249(13)$ \\
Ni1-S2 & $2.2157(18)$ & $2.2194(14)$ \\
S1-C1 & $1.697(7)$ & $1.710(5)$ \\
S2-C1 & $1.705(7)$ & $1.703(5)$ \\
C1-O1 & $1.306(9)$ & $1.306(6)$ \\
& & \\
S2-Ni-S1 & $79.53(6)$ & $79.27(5)$ \\
S2-C1-S1 & $113.1(4)$ & $112.3(3)$ \\
C1-S1-Ni1 & $83.7(2)$ & $84.04(18)$ \\
C1-S1-Ni1 & $83.7(2)$ & $84.4(2)$ \\
\hline
\end{tabular}

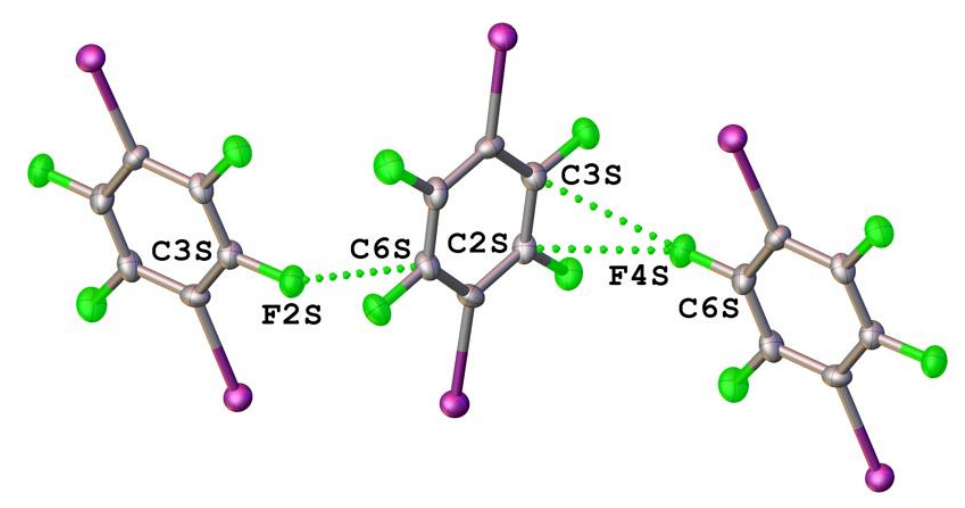

Fig. S1. $L P-\pi$ h contacts (dotted lines) between $1,4-F I B s$ in the crystal structure of $\mathbf{1} \cdot 2(1,4-\mathrm{FIB})$.

In the XRD structure of $\mathbf{1} \cdot 2(1,4-\mathrm{FIB})$, short contacts between F-atoms and C-atoms of neighboring 1,4-FIBs were observed (Fig. S1). Inspection of the geometric characteristics of these noncovalent interactions (contact C6S $\cdots$ F2S: distance C6S-F2S 2.921(8) vs. $\Sigma_{\mathrm{vdW}} \mathrm{C}+\mathrm{F} 3.17 \AA$, $\angle \mathrm{C} 6 \mathrm{~S}-\mathrm{F} 2 \mathrm{~S}-\mathrm{C} 3 \mathrm{~S} 152.9(4)$ and $\angle \mathrm{C} 5 \mathrm{~S}-\mathrm{C} 6 \mathrm{~S}-\mathrm{F} 2 \mathrm{~S} 110.6(5)^{\circ}$; contact (C2S,C3S) $\cdots \mathrm{F} 4 \mathrm{~S}$ : distances

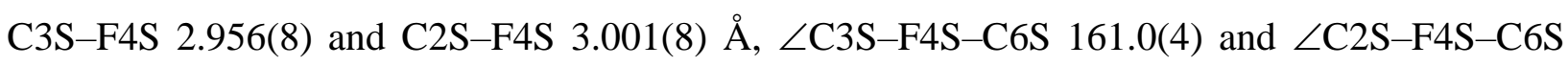
$155.8(4)^{\circ}, \angle \mathrm{C} 2 \mathrm{~S}-\mathrm{C} 3 \mathrm{~S}-\mathrm{F} 4 \mathrm{~S} 74.8(4)$, and $\left.\angle \mathrm{C} 3 \mathrm{~S}-\mathrm{C} 2 \mathrm{~S}-\mathrm{F} 4 \mathrm{~S} 78.5(4)^{\circ}\right)$ indicate that these contacts can be attributed to $\mathrm{LP}-\pi \mathrm{h}$ interactions. In these contacts, an electropositive area of the perfluoroarene ring forms a contact with electron-donating F-atoms. 


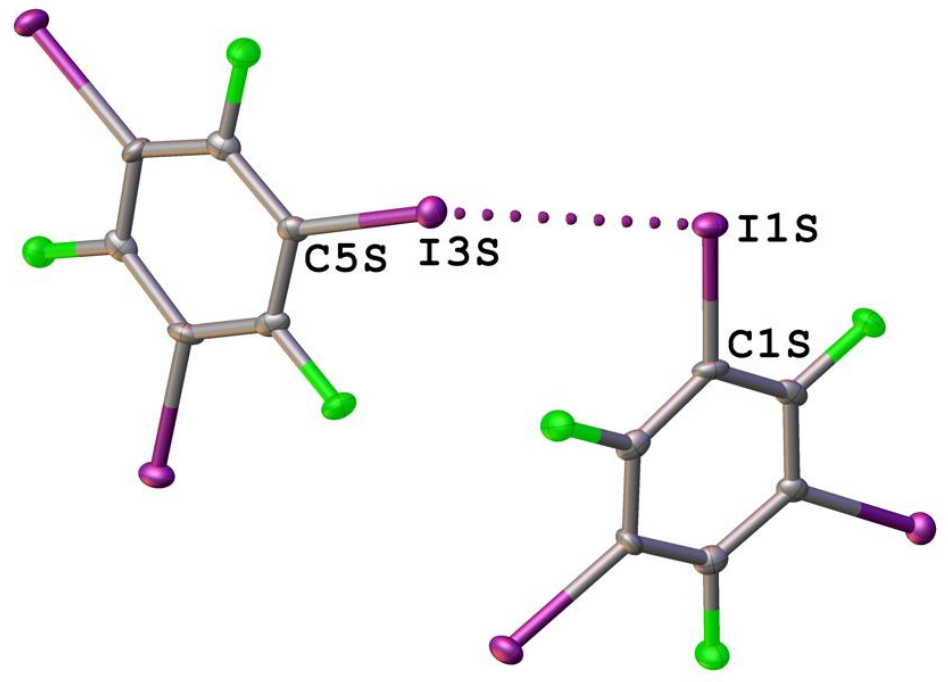

Fig. S2. XB (dotted lines) between 1,3,5-FIBs in the XRD structure of 1·2(1,3,5-FIB).
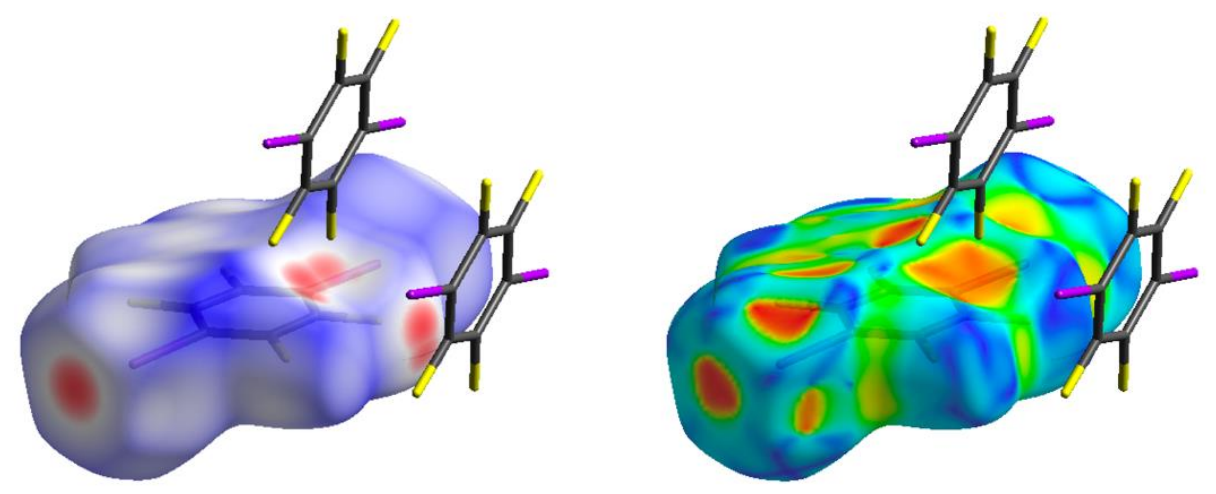

Fig. S3. Hirshfeld surfaces mapped with $d_{\text {norm }}$ over the range -0.21 (red) to 1.1 (blue) (left) and shape index S, mapped from -1.0 (concave hollows; red) $\rightarrow 0.0$ (minimal saddle; green) $\rightarrow+1.0$ (convex bumps; blue)(right) for 1,4-FIB in the XRD structure of $1 \cdot 2(1,4-\mathrm{FIB})$. The view indicates formation of the $\mathrm{LP}-\pi$-hole interaction between $1,4-\mathrm{FIB}$ molecules. 

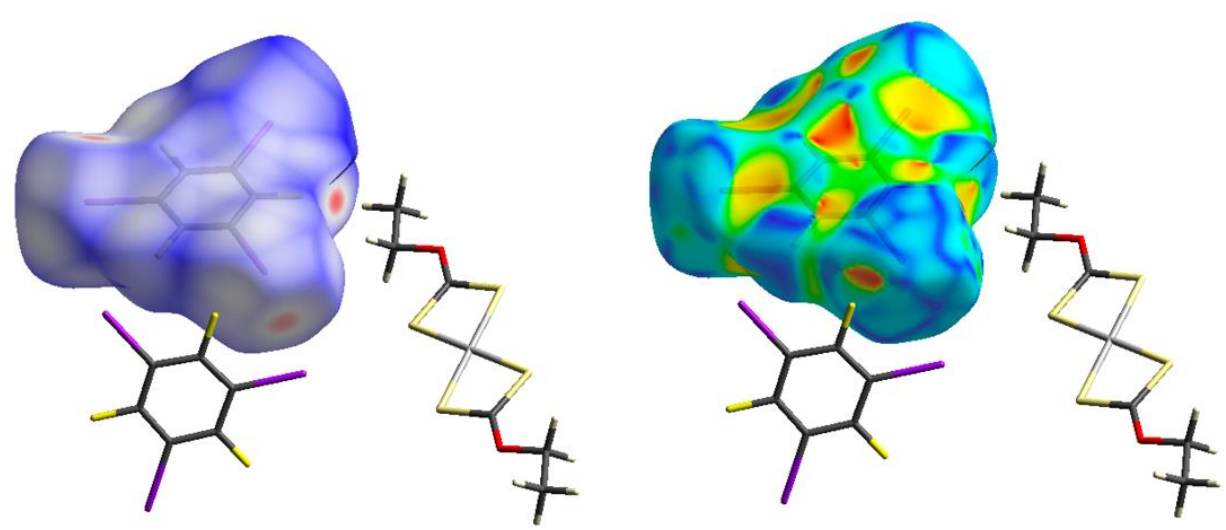

Fig. S4. Hirshfeld surfaces mapped with $d_{\text {norm }}$ over the range -0.2 (red) to 1.0 (blue) (left) and shape index S, mapped from -1.0 (concave hollows hollows; red) $\rightarrow 0.0$ (minimal saddle; green) $\rightarrow+1.0$ (convex bumps; blue)(right) for 1,3,5-FIB in the XRD structure of $1 \cdot 2(1,4-\mathrm{FIB})$. The view indicates formation of the I $\cdots$ I XB between two 1,3,5-FIB molecules and the H $\cdots$ F HB between 1,3,5-FIB and $\mathrm{CH}_{3}$ fragment in $\mathbf{1}$. 